\title{
Consumo alimentar de mulheres sobreviventes de câncer de mama: análise em dois períodos de tempo
}

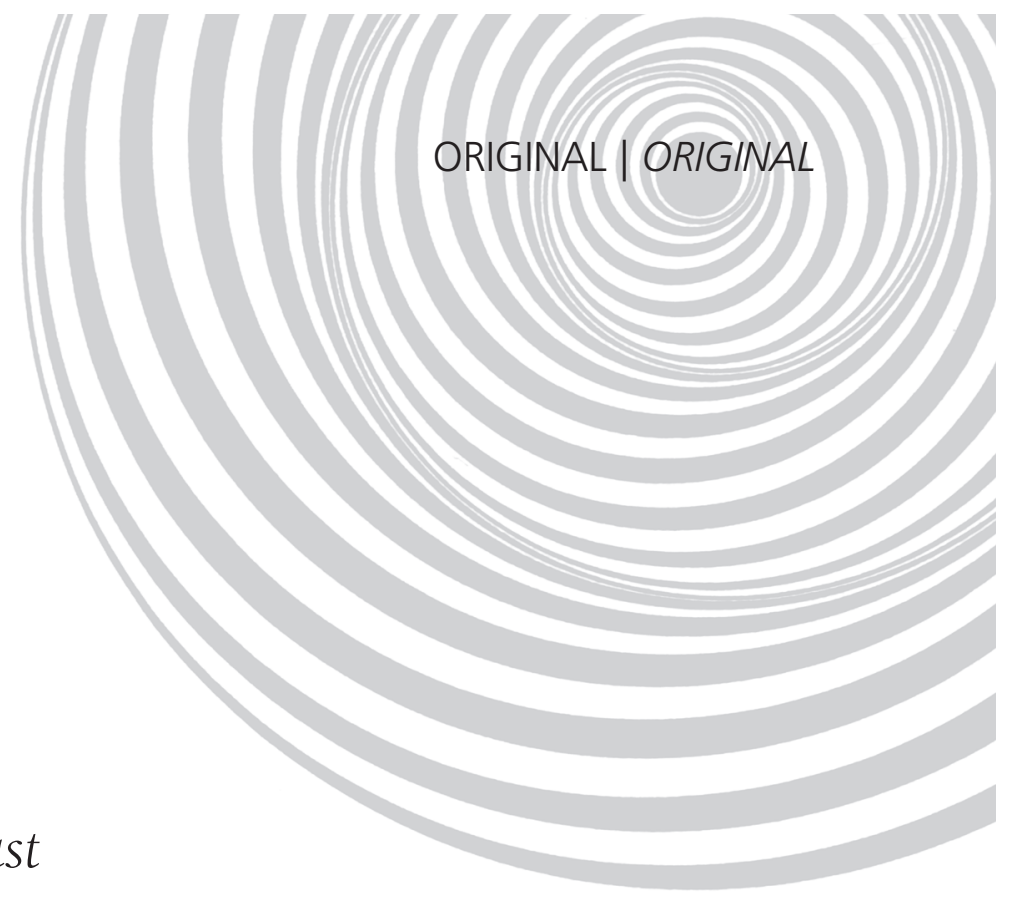

\section{Food intake of women survivors of breast cancer: analysis in two time periods}

Helena Alves de Carvalho SAMPAIO'

Daianne Cristina ROCHA'

Maria Olganê Dantas SABRY'

Luiz Gonzaga Porto PINHEIRO'

RE S U M O

\section{Objetivo}

Comparar o consumo alimentar de dois grupos de mulheres portadoras de câncer de mama atendidas em dois períodos distintos: 1999-2004 e 2005-2009.

\section{Métodos}

Estudo documental, com abordagem descritiva e comparativa. Foram coletados dados de identificação e de ingestão alimentar nas fichas de 154 pacientes portadoras de câncer de mama atendidas em serviços de referência de Fortaleza (CE): 66 no período de 1999-2004 e 88 no de 2005-2009. Foram considerados como integrantes do hábito os alimentos consumidos pelo menos quinzenalmente por, no mínimo, 50\% das pessoas. Calculou-se a prevalência de consumo de alimentos, sendo os padrões dos dois períodos comparados pela aplicação dos testes Qui-quadrado ou exato de Fisher, e adotou-se $p<0,05$ como nível de significância.

\section{Resultados}

Os hábitos alimentares dos dois grupos foram semelhantes, exceto quanto ao maior consumo de leite e de cenoura e menor consumo de manga e caju, além de uma tendência ao aumento no consumo de óleo de soja, observada no grupo atendido mais recentemente. O aumento de leite e de cenoura pode configurar proteção contra a recidiva da doença, mas a redução do consumo das frutas citadas pode significar maior risco: aliás, foram baixas as prevalências de consumo de frutas e hortaliças pelos dois grupos de mulheres.

\section{Conclusão}

Os achados apontam para uma lacuna na abordagem educativa das pacientes tanto no passado como na atualidade, o que comprova a necessidade de incentivar maior consumo e variedade de hortaliças e frutas e menor consumo de cereais refinados, gorduras, açúcar e refrigerantes.

Termos de indexação: Câncer de mama. Dieta. Nutrição.

\footnotetext{
1 Universidade Estadual do Ceará, Centro de Ciências da Saúde, Curso de Nutrição. Av. Paranjana, 1700, Itaperi, 60740-903, Fortaleza, CE, Brasil. Correspondência para/Correspondence to: HAC SAMPAIO. E-mail: <dr.hard@terra.com.br>.
} 


\section{A B S T R A C T}

\section{Objective}

The aim of this study is to compare the dietary intake of two groups of women with breast cancer treated on different periods: 1999-2004 and 2005-2009.

\section{Methods}

This descriptive and comparative documental study collected data from the medical records of 154 patients with breast cancer treated in reference centers of Fortaleza (CE), Brazil. The following variables were studied: food intake and characterization. Sixty-six women were studied from 1999-2004 and 88 from 2005-2009. Foods consumed at least every 15 days by not less than $50 \%$ of the women were considered part of their eating habits. The prevalence of the foods consumed was calculated, and the standards of the two periods compared by the Chi-square or Fisher's exact test. The significance level was set at $5 \%(p<0.05)$.

\section{Results}

The dietary habits of both groups were similar but the group treated more recently consumed more milk, less carrots, mangos and cashews, and tended to consume more soybean oil. Higher intakes of milk and carrots can provide protection against recurrence, but lower consumption of fruits may increase risk.

\section{Conclusion}

These findings indicate that patients are not being properly advised, both in the past and today. Patients need to be encouraged to consume more vegetables and fruits in volume and variety, and less refined grains, fats, sugar and soda.

Indexing terms: Breast neoplasms. Diet. Nutrition.

\section{N T R O D U Ç Ã O}

Com as crescentes industrialização e globalização, as neoplasias malignas ganharam importância crescente no perfil de mortalidade mundial; no Brasil, elas ocupam segundo lugar como causa de óbito' 1 . Dentre as neoplasias, as que atingem maior número de pessoas, excetuando-se os tumores de pele não melanoma, são as de próstata e de pulmão no sexo masculino, e os cânceres de mama e colo do útero no sexo feminino ${ }^{2}$.

O câncer de mama é o mais frequente e o de maior incidência entre as mulheres brasileiras ${ }^{3}$. Segundo o Instituto Nacional de Câncer ${ }^{2}$, previsão que abrange o biênio 2012-2013 prevê 53 mil novos casos de câncer de mama no Brasil.

Vários são os fatores de risco para o aparecimento do câncer de mama4 ${ }^{4}$ nuliparidade, idade tardia ao primeiro parto, menarca precoce, menopausa tardia, sedentarismo, dieta e obesidade ${ }^{4}$. Dentre eles, os fatores dietéticos contribuem na etiologia de 30 a $40 \%$ dos casos $^{5}$. Aspectos nutricionais - seja estado nutricional, seja alimentação habitual - são apontados tanto como protetores como desencadeadores do câncer de mama ${ }^{5}$.

Quanto à prevenção da doença, a literatura aponta que se deve evitar excesso de peso e ganho ponderal na vida adulta, bem como excesso de gordura corporal e abdominal6-8. Uma dieta com consumo elevado de frutas, grãos integrais e hortaliças, em que se aliam nutrientes antioxidantes, fibras e compostos fitoquímicos, tem ação preventiva ${ }^{6,9}$. São considerados risco para a doença tanto o baixo consumo dos alimentos supracitados como o excesso de ingestão de alimentos ricos em alguns tipos de gordura, como saturada, poli-insaturada e trans, tais como carnes vermelhas, frituras, molhos com maionese, leite integral e derivados, bacon, presunto, salsicha, linguiça e mortadela ${ }^{10}$.

No âmbito terapêutico, os antioxidantes aumentam a eficácia do mecanismo de ação das drogas antineoplásicas, culminando em redução do tamanho do tumor com produção de menos efeitos colaterais, aumentando o tempo de sobrevida e melhorando a qualidade de vida ${ }^{11}$. Os com- 
postos fitoquímicos também possuem vários efeitos benéficos devidos à atividade antioxidante e aos hormônios gonadais, diminuindo o risco de progressão da neoplasia mamária9,12.

Vários estudos evidenciam que mulheres sobreviventes de câncer de mama precisam ter o mesmo perfil nutricional (estado nutricional e dieta) recomendado para prevenção da doença a fim de prevenir sua recorrência9,13. Sintomas como anorexia, saciedade precoce, mudanças no paladar e no olfato e distúrbios do trato gastrintestinal são efeitos colaterais comuns do tratamento do câncer e podem levar à ingestão mais inadequada de nutrientes durante sua vigência ${ }^{14}$.

Nessa perspectiva, tanto em serviços de atendimento preventivo da doença como em serviços de tratamento, torna-se fundamental a inclusão de aconselhamento nutricional às mulheres atendidas que promova um bom estado nutricional, incentive a ingestão de alimentos protetores e restrinja o consumo de alimentos de risco. Infelizmente, há poucas publicações nacionais que avaliem o desenvolvimento dessas ações educativas e sua taxa de sucesso.

Analisando a situação de forma empírica, parece haver uma preocupação maior com aspectos preventivos relacionados à realização de exames clínicos, laboratoriais, e de imagem (ultrassonografia e mamografia), mas pouca ação no que tange à educação nutricional.

Assim, o presente estudo objetiva avaliar e comparar o padrão alimentar de mulheres portadoras de câncer de mama, atendidas no período de 1999 a 2004 e no período de 2005 a 2009, em serviços de referência na prevenção e tratamento dessa afecção. Hipotetiza-se que haja uma insuficiente abordagem educativa nutricional e que um consumo dietético de risco, se presente, vai se reproduzindo ao longo do tempo, sem permitir detectar melhora da qualidade alimentar, podendo, ainda, haver piora dietética devido à potencial influência negativa da globalização e da mídia.

\section{MÉ T O D O S}

Trata-se de um estudo caracterizado como documental, com abordagem descritiva e comparativa. Utilizou-se o banco de dados da pesquisa "Alterações do gasto energético basal, composição corporal e padrão alimentar de portadores de doenças crônicas", financiada pela Fundação Cearense de Apoio ao Desenvolvimento Científico (FUNCAP).

Foram analisadas todas as fichas de mulheres avaliadas portadoras de câncer de mama, atendidas em três serviços de referência localizados na cidade de Fortaleza (CE), totalizando 154 fichas: 66 de mulheres atendidas no período de 1999 a 2004 (Período 1) e 88 de mulheres atendidas no período de 2005 a 2009 (Período 2): todas em terapia antineoplásica, ainda que algumas estivessem em sua finalização.

Os dados avaliados foram os sociodemográficos e os referentes ao consumo alimentar. Foram ainda compilados, nas fichas, dados referentes ao Índice de Massa Corporal (IMC) de cada paciente. O IMC foi avaliado quanto à presença de excesso ponderal, adotando-se a classificação da World Health Organization ${ }^{15}$ : valores iguais ou superiores a $25 \mathrm{~kg} / \mathrm{m}^{2}$ equivalem à presença de excesso de peso.

Para obtenção dos dados referentes à ingestão alimentar, foram utilizadas as informações obtidas por meio de um questionário de Frequência Alimentar Qualitativo. O instrumento utilizado e anexado às fichas avaliadas abrangia 132 itens, distribuídos segundo grupo alimentar (leite e derivados; carnes e ovos; cereais e leguminosas; hortaliças; frutas; açúcares e doces; óleos e gorduras; miscelânea), com discriminação das seguintes frequências de consumo: diária, quatro a seis vezes por semana, uma a três vezes por semana, três vezes por mês, duas vezes por mês, uma vez por mês, menos do que uma vez por mês e nunca.

É necessário ressaltar que, à época do início do estudo, não estava disponível um questionário de frequência alimentar local que tivesse 
sido validado. O questionário utilizado na presente pesquisa, portanto, foi desenvolvido pelos autores, como parte de outra investigação, também com foco na inter-relação, no ano anterior ao início do presente estudo, quando foram listados todos os alimentos comercializados na capital, em supermercados e feiras livres. No momento da aplicação do questionário, era, ainda, indagado se a respondente consumia algum alimento não citado, e, caso a resposta fosse afirmativa, acrescentava-se o item ao questionário.

Na ausência de um referencial teórico consensual que defina o que seja um consumo alimentar habitual de um grupo, estabeleceu-se como ponto de corte os alimentos consumidos pelo menos duas vezes no mês por, no mínimo, $50 \%$ das pessoas. Para comparação dos dados, foram também incluídos alimentos que em um dos períodos tivesse prevalência de consumo inferior a $50 \%$, desde que no outro período atingisse tal percentual.

As médias de renda familiar e IMC das mulheres do P1 e P2 foram comparadas através do test $t$ de Student, após aplicação do teste de normalidade de Levene. A escolaridade, a presença de excesso ponderal e a situação alimentar dos dois grupos foram comparadas através do teste Qui-quadrado. Para todos os testes, foi adotado $p<0,05$ como nível de significância. Como ferramenta operacional para essas análises, foi utilizado o software Epi Info ${ }^{16}$, versão 6.0 e o Statistical Package for the Social Sciences (SPSS) Statistics $17.0^{17}$.

O trabalho original foi submetido e aprovado pelo Comitê de Ética em Pesquisa com Seres Humanos da Instituição CAAE - 0041.0.038.000-08. Este estudo foi delineado segundo a resolução 196/96, que orienta a realização de pesquisas com seres humanos. Nas fichas avaliadas, constavam os Termos de Consentimento Livre e Esclarecido para participação na pesquisa. Para utilização dos dados, foi assinado um Termo de Fiel Depositário.

\section{RESULTADOS}

A idade média das participantes do estudo foi de Média $(M)=51,63$, Desvio-Padrão $(D P)=10,69$ anos, sendo semelhante à das integrantes do $P 1, M=51,21, D P=11,52$ anos, e do $P 2, M=51,39, D P=10,43$.

A renda mensal média encontrada foi de $M=2,88, D P=2,41$ salários-mínimos. A renda média mensal das integrantes do $P 1 \quad(M=3,66$, $\mathrm{DP}=2,87)$ era um pouco mais elevada do que a das integrantes do P2 $(M=2,26, D P=1,77)$ $(p=0,001)$. A maioria das pacientes tinha escolaridade de até, no máximo, ensino fundamental completo $(65,58 \%)$, embora cerca de um quarto delas $(25,32 \%)$ tivesse o ensino médio completo. Essa distribuição foi similar, considerando as respondentes do P1, com $65,15 \%$ com ensino fundamental completo e $24,00 \%$ com ensino médio completo, e as respondentes do P2, com 65,91\% apresentando ensino fundamental completo e $26,14 \%$, ensino médio completo. Considerando a presença de ensino fundamental completo, não houve diferença entre as mulheres do P1 e do P2 $(p=0,922)$.

O IMC médio do grupo foi $M=27,14$, $D P=4,64 \mathrm{~kg} / \mathrm{m}^{2}$, sendo $M=27,04, D P=4,70 \mathrm{~kg} / \mathrm{m}^{2}$ entre as participantes do $\mathrm{P} 1$ e $M=27,23$, $D P=4,62 \mathrm{~kg} / \mathrm{m}^{2}$ entre as participantes do $P 2$, sem diferença estatística $(p=0,805)$. A presença de excesso de peso foi semelhante: $62,12 \%$ das mulheres do P1 e $65,9 \%$ das do P2 $(p=0,627)$.

Foram avaliados os alimentos principalmente proteicos de origem animal, carnes/ovos e leite e derivados consumidos pelas pacientes segundo o período investigado. Observa-se um aumento significante na prevalência de ingestão de leite tipo B/C no P2. O grupo de leite e derivados não integrava o hábito das mulheres do P1, segundo o ponto de corte de consumo habitual aqui adotado. Dentre os demais alimentos, o consumo mais prevalente em ambos os períodos corresponde à carne de frango e à bovina, seguidas pelos pescados (Tabela 1). 
Quanto aos resultados referentes ao consumo de cereais e leguminosas: novamente observa-se que não houve alterações no consumo alimentar entre os dois períodos. Os sete alimentos identificados nesse grupo alimentar tiveram alta prevalência de consumo, com destaque para o arroz e feijão (Tabela 2).

Quanto ao grupo das hortaliças, o consumo de cenoura foi maior no P2. Observa-se que, dentre os doze alimentos, excluindo-se batata inglesa, mais associada a tubérculo, e outros quatro alimentos citados, que habitualmente são utilizados como tempero (cebola, cebolinha, coentro e pimentão), restam apenas sete hortaliças habitualmente consumidas, duas delas folhosas: alface e repolho (Tabela 3).

No grupo das frutas, observa-se, no P2, redução no consumo de caju e manga. Houve referência a 11 frutas, mas a maioria com prevalência de consumo mais baixa do que o detectado com integrantes de outros grupos alimentares, à exceção da banana, laranja e mamão (Tabela 4).

Em relação aos grupos de açúcares, gorduras e outros, não houve diferença de consumo nos dois períodos, mas houve uma tendência ao aumento de ingestão de óleo de soja $(p=0,050)$ no P2. Foram citados sete alimentos, com maior destaque para colorau, óleo de soja, café, açúcar refinado e margarina (Tabela 5).

Tabela 1. Prevalência de consumo de alimentos fontes de proteínas de origem animal entre as mulheres avaliadas, segundo o período investigado e análise estatística. Fortaleza (CE), 1999 a 2009.

\begin{tabular}{lcccc}
\hline Alimentos & P1 (\%) & P2 (\%) & $\chi^{2}$ & $p$ \\
\hline Carne de frango & 96,97 & 95,45 & 0,23 & 0,631 \\
Carne de vaca $^{*}$ & 89,39 & 87,50 & 0,13 & 0,717 \\
Fígado* $^{*}$ & 43,49 & 50,00 & 0,56 & 0,456 \\
Ovo de galinha $_{\text {Peixe }}^{69,70}$ & 61,36 & 1,15 & 0,283 \\
Leite de vaca tipo B/C $^{*}$ & 77,27 & 75,00 & 0,11 & 0,744 \\
Leite de vaca em pó $^{*}$ & 45,45 & 67,05 & 7,21 & 0,007 \\
Queijo coalho $^{*}$ & 43,94 & 53,41 & 1,35 & 0,245 \\
\hline
\end{tabular}

*Alimentos não faziam parte do hábito alimentar no P1.

P1: Período 1 (1999 a 2004); P2: Período 2 (2005 a 2009).
Tabela 2. Prevalência de consumo do grupo alimentar de cereais e leguminosas entre as mulheres avaliadas, segundo o período investigado e análise estatística. Fortaleza (CE), 1999 a 2009

\begin{tabular}{lcccc}
\hline Alimentos & P1 (\%) & P2 (\%) & $\chi^{2}$ & $p$ \\
\hline Arroz & 96,97 & 98,86 & 0,71 & 0,400 \\
Biscoito salgado & 84,85 & 76,14 & 1,78 & 0,182 \\
Cuscuz & 75,76 & 76,14 & 0,00 & 0,956 \\
Farinha de mandioca & 68,18 & 73,86 & 0,60 & 0,440 \\
Feijão & 98,48 & 98,60 & 0,04 & 1,000 \\
Macarrão & 87,88 & 87,50 & 0,01 & 0,944 \\
Pão & 86,36 & 89,77 & 0,42 & 0,515 \\
\hline
\end{tabular}

P1: Período 1 (1999 a 2004); P2: Período 2 (2005 a 2009).

Tabela 3. Prevalência de consumo do grupo alimentar de hortaliças entre as mulheres avaliadas, segundo o período investigado e análise estatística. Fortaleza (CE), 1999 a 2009.

\begin{tabular}{lcccc}
\hline Alimentos & P1 (\%) & P2 (\%) & $\chi^{2}$ & $p$ \\
\hline Alface & 59,09 & 67,05 & 1,03 & 0,310 \\
Batata inglesa & 83,33 & 90,91 & 2,00 & 0,157 \\
Beterraba & 66,67 & 67,05 & 0,00 & 0,960 \\
Cebola & 95,45 & 100,00 & 4,08 & 0,077 \\
Cebolinha & 92,42 & 98,86 & 4,18 & 0,084 \\
Cenoura & 77,27 & 89,77 & 4,48 & 0,034 \\
Chuchu & 84,85 & 78,41 & 1,02 & 0,312 \\
Coentro & 92,42 & 97,73 & 2,44 & 0,139 \\
Jerimum & 50,00 & 53,41 & 0,18 & 0,675 \\
Pimentão & 83,33 & 89,77 & 1,38 & 0,239 \\
Repolho & 50,00 & 53,41 & 0,18 & 0,675 \\
Tomate & 92,42 & 95,45 & 0,63 & 0,499 \\
\hline
\end{tabular}

P1: Período 1 (1999 a 2004); P2: Período 2 (2005 a 2009).

Tabela 4. Prevalência de consumo do grupo alimentar das frutas entre as mulheres avaliadas, segundo o período investigado e análise estatística. Fortaleza (CE), 1999 a 2009.

\begin{tabular}{lcccc}
\hline Alimentos & P1 (\%) & P2 (\%) & $\chi^{2}$ & $p$ \\
\hline Acerola & 71,21 & 68,18 & 0,16 & 0,686 \\
Banana & 89,39 & 95,45 & 2,09 & 0,207 \\
Caju* $^{*}$ & 50,00 & 28,41 & 7,49 & 0,006 \\
Goiaba & 65,15 & 77,27 & 2,75 & 0,097 \\
Laranja & 80,30 & 85,23 & 0,65 & 0,419 \\
Maçã & 63,64 & 69,32 & 0,55 & 0,458 \\
Mamão & 81,82 & 88,64 & 1,43 & 0,231 \\
Manga* & 62,12 & 42,05 & 6,08 & 0,014 \\
Maracujá & 65,15 & 72,73 & 1,02 & 0,312 \\
Melancia* & 53,03 & 48,86 & 0,26 & 0,609 \\
Uva & 56,06 & 51,14 & 0,37 & 0,544 \\
\hline
\end{tabular}

*Alimentos não faziam parte do hábito alimentar no P2.

P1: Período 1 (1999 a 2004); P2: Período 2 (2005 a 2009). 
602 | HAC SAMPAIO et al.

Tabela 5. Prevalência de consumo dos grupos alimentares de açúcares, gorduras e outros entre as mulheres avaliadas, segundo o período investigado e análise estatística. Fortaleza (CE), 1999 a 2009.

\begin{tabular}{|c|c|c|c|c|}
\hline Alimentos & P1 (\%) & $\mathrm{P} 2(\%)$ & $\chi^{2}$ & $p$ \\
\hline Açúcar refinado & 81,82 & 78,41 & 0,27 & 0,601 \\
\hline Margarina & 71,21 & 78,41 & 1,05 & 0,305 \\
\hline Óleo de soja & 85,84 & 94,32 & 3,85 & 0,050 \\
\hline Café & 86,36 & 86,36 & 0,00 & 1,000 \\
\hline Caldo de galinha industrializado* & 45,45 & 53,41 & 0,95 & 0,328 \\
\hline Colorau & 90,91 & 93,18 & 0,27 & 0,602 \\
\hline Refrigerante & 63,64 & 57,95 & 0,51 & 0,475 \\
\hline
\end{tabular}

*Alimentos não faziam parte do hábito alimentar no P1.

P1: Período 1 (1999 a 2004); P2: Período 2 (2005 a 2009).

\section{I S C U S S Ã O}

Os hábitos alimentares são influenciados por fatores socioeconômicos e culturais que determinam o que, com quem, quando e quanto se come $^{18}$. Nas últimas décadas, o processo de globalização passou a influenciar o consumo alimentar e a gerar certa uniformidade de hábitos no mundo todo, com o abandono de alimentos tradicionais de uma região ${ }^{19}$.

Muitos desses alimentos da modernidade, mais especificamente alimentos industrializados com alta densidade energética, têm sido associados ao surgimento de doenças crônicas, sendo importante que ações de educação nutricional sejam realizadas a fim de colocar alimentos tradicionais e saudáveis de volta ao prato da população ${ }^{20}$.

O presente estudo apresenta um aspecto inédito: comparar o consumo de dois grupos de mulheres portadoras de câncer de mama - um atendido no período de 1999 a 2004 e outro atendido de 2004 a 2009 -, ambos nas mesmas instituições. Tal comparação permite identificar os alimentos que foram mantidos, introduzidos ou retirados do consumo alimentar habitual desse grupo populacional.

Estudos, inclusive de base populacional, têm evidenciado piora do hábito alimentar de diferentes grupos populacionais ao longo do tempo. Pesquisa realizada em 2010 pelo Ministério da Saúde revelou que o padrão alimentar brasileiro também mudou para pior. O feijão, que tradicionalmente fazia o famoso par com o arroz, perdeu espaço na mesa dos brasileiros: em 2006, $71,9 \%$ da população comiam a leguminosa ao menos cinco vezes na semana; em 2010, a média caiu para $65,8 \%$. Também foi descoberto que o consumo de gorduras é elevado no País, e, principalmente, nas regiões Sul, Sudeste e Centro-Oeste, onde ocorrem as maiores incidências de câncer de mama. Os refrigerantes e sucos artificiais ganharam espaço na preferência dos brasileiros: ao todo, $76,0 \%$ dos adultos bebem esses produtos pelo menos uma vez por semana, e $27,9 \%$ cinco vezes ou mais durante o mesmo período. Entretanto, o consumo de alimentos ricos em fatores de proteção contra o câncer, tais como frutas, verduras, legumes e cereais, aumentou, embora ainda seja baixo: 18,9\% disseram consumir cinco porções diárias desses alimentos, mais do que o dobro do percentual registrado em $2006^{21}$.

Comparativamente, no grupo avaliado, percebe-se que os hábitos alimentares foram semelhantes entre as mulheres do P1 e as do P2, com exceção do maior consumo de leite e de cenoura e menor consumo de manga e caju detectado entre as do P2. No entanto, percebe-se que é um hábito relativamente monótono, com apenas 45 alimentos, dentre os 132 elencados e disponíveis na região.

Embora não tenha sido investigado o motivo da maior ingestão de leite, pode ser levantada a hipótese de que as mulheres tenham sido orientadas para tal consumo, pois agentes quimiote- 
rápicos levam à diminuição da função ovariana, acarretando uma menopausa precoce e, consequentemente, um risco aumentado de desenvolverem osteopenia ou osteoporose ${ }^{22}$. Por outro lado, teria sido mais interessante um aumento de frequência de ingestão de leite desnatado, dada a implicação das gorduras no aumento do risco de recidiva da doença ${ }^{10}$.

A lista de frutas consumidas é relativamente grande, um dado positivo para o grupo, embora a quantidade ingerida deva ser avaliada em estudos adicionais. A redução da ingestão de caju e manga é inesperada, pois são frutas regionais. Mesmo sendo sazonais, como o levantamento de consumo ocorreu ao longo do ano todo em ambos os períodos, a sazonalidade não deve ter sido fator influenciador da realidade encontrada. Um componente educativo importante junto a esse tipo de paciente é o incentivo ao consumo de frutas regionais devido ao menor custo pela maior disponibilidade.

As hortaliças são pouco consumidas, pois muitas dentre as citadas são feculentas (tubérculos e raízes), e muitas são utilizadas como condimentos em preparações diversas, como cebolinha, pimentão e coentro. As recomendações de ingestão de hortaliças são referentes a hortaliças não feculentas ${ }^{20}$, de forma que os dois grupos atendidos necessitavam de orientações quanto a esse grupo alimentar.

O consumo de cereais, açúcar e refrigerantes, além dos tubérculos, que atingem um total de 10 alimentos, representa $22,22 \%$ do total de alimentos ingeridos e pode configurar risco para as mulheres avaliadas, uma vez que essa clientela costuma ganhar peso com a terapêutica antineoplásica ${ }^{23}$.

Em estudo realizado por Kwan et al..$^{24} \mathrm{com}$ 1901 pacientes com câncer de mama, constatou-se que o aumento da adesão a hábitos alimentares mais saudáveis, com uma dieta rica em frutas, verduras, cereais integrais, aves, peixe e produtos lácteos com baixo teor de gordura, estava associado a uma diminuição da mortalidade geral $(p=0,02)$, ao passo que o aumento do consumo de dieta típica do padrão ocidental (alto consumo de carnes vermelhas e processadas, cereais refinados, produtos lácteos com alto teor de gordura e doces) estava associado $(p=0,05)$ a um risco crescente de morte.

Também McEligot et al. ${ }^{25}$, em estudo realizado com 516 mulheres diagnosticadas com câncer de mama, observaram que a redução da gordura corporal e o aumento de fibras, vegetais e frutas na dieta melhoraram a sobrevida após o diagnóstico da doença.

Um aspecto que deve ser debatido é a menor renda familiar encontrada entre as mulheres do P2. No entanto, esse fato não teve repercussão negativa no consumo alimentar, pois nesse grupo houve até elevação na prevalência de ingestão de alguns alimentos. Assim, houve aumento na prevalência de ingestão de laticínios em relação ao P1, e, considerando as hortaliças e frutas, mesmo com prevalências não tão elevadas, no P2 houve aumento do consumo desses itens, embora com significância estatística apenas para cenoura. No caso das frutas, a redução de caju e manga, já citada, no P2, também não deve se associar à renda, pois, quando presentes na safra, o custo de aquisição é baixo.

Dois outros aspectos de caracterização do grupo que devem ser enfocados são a escolaridade e o estado nutricional, determinado pelo IMC. Independentemente do período avaliado, as mulheres exibiram escolaridade, média de IMC e prevalência de excesso ponderal similares, descartando a influência dessas variáveis na realidade encontrada.

Como já referido, sobreviventes de câncer de mama necessitam manter um consumo alimentar saudável com o intuito de prevenir recidiva da doença ${ }^{13}$. Os grupos avaliados, em diferentes períodos de tempo, ao menos qualitativamente, demonstraram, confirmando a hipótese levantada, uma constância de comportamento alimentar, em que erros detectados no passado também estiveram presentes em dias mais atuais. Assim, observou-se uma similaridade de fontes proteicas oriundas de carnes brancas e vermelhas que deve ser avaliada quantitativamente a fim 
de se verificar se há um excesso de ingestão lipídica delas proveniente. Quanto aos cereais, constatou-se a ausência de integrais, o que pode limitar o consumo adequado de fibras. Verificou-se, ainda, a necessidade de se elevarem as prevalências de consumo de hortaliças e frutas, incorporando, por exemplo, outros alimentos disponíveis, como acelga, maxixe, quiabo, berinjela, melão, tangerina, cajá, sapoti e ata. O aumento de prevalência de ingestão e de variedade elevará a oferta de vitaminas, minerais, fibras e fitoquímicos, como recomendado pelas diretrizes mundiais de prevenção da doença e de sua recidiva ${ }^{13}$. A elevação de ingestão de margarina (não significante) e de óleo de soja também deve ser investigada quanto à quantidade a fim de se constatar presença ou ausência de excesso de ingestão de gorduras, componente de risco para a doença ${ }^{13}$.

Todos os aspectos apontados constituem um alerta para que a equipe de saúde delineie ações educativas para essas mulheres, visando a elevar o consumo de alimentos saudáveis e a reduzir o consumo de alimentos associados ao risco para a doença, e, no caso, para sua recidiva.

Logicamente, o estudo apresenta algumas limitações: uma delas refere-se ao fato de ter sido abordada apenas a frequência alimentar qualitativa e não a quantidade dos alimentos consumidos, aspecto que deverá ser enfocado em estudos posteriores. Outra limitação é relativa ao ponto de corte utilizado para definir o consumo alimentar habitual, que foi a ingestão do alimento por pelo menos $50 \%$ dos entrevistados, pelo menos duas vezes por mês. Na literatura, os percentuais populacionais e as frequências de ingestão para definir consumo alimentar habitual são variados, englobando $50-70 \%$ das pessoas e consumo diário, semanal ou mensal26-30. Ao considerar uma ingestão ao menos quinzenal, pode ter havido superestimação do consumo cotidiano dos alimentos citados.

\section{O N CLUS Ã O}

O consumo alimentar habitual identificado tanto aponta para uma potencial lacuna na abor- dagem educativa pregressa e atual dessa clientela como para os aspectos que deverão ser priorizados em ações de intervenção. Especificamente, deverá haver um incentivo ao maior consumo de hortaliças e frutas, considerando-se uma maior variedade delas, e à diminuição do consumo de cereais refinados, gorduras, açúcar e refrigerante.

\section{COLABORADORES}

HAC SAMPAIO colaborou na concepção do estudo, análise e interpretação dos dados, redação do estudo e supervisão geral do estudo. DC ROCHA colaborou na coleta, análise e interpretação dos dados e na redação do estudo. MOD SABRY colaborou na análise e interpretação dos dados e redação do estudo. LGP PINHEIRO colaborou na redação e na supervisão geral do estudo.

\section{REFERÊ NCIAS}

1. Zandonai AP. A busca de evidências para os fatores de risco alimentares do câncer colorretal: revisão integrativa da literatura [dissertação]. Ribeirão Preto: Universidade de São Paulo; 2010.

2. Brasil. Ministério da Saúde. Instituto Nacional de Câncer. Estimativa 2012: incidência de câncer no Brasil [Internet]. Rio de Janeiro: INCA; 2011 [acesso 2011 dez 27]. Disponível em: <http://www.inca. gov.br/estimativa/2012/estimativa20122111.pdf>.

3. Moraes AB, Zanini RR, Turchiello MS, Riboldi J, Medeiros LR. Estudo da sobrevida de pacientes com câncer de mama atendidas no hospital da Universidade Federal de Santa Maria, Rio Grande do Sul, Brasil. Cad Saúde Pública [Internet]. 2006 [acesso 2011 jun 27]; 22(10):2219-28. Disponível em: <http://www.scielo.br/pdf/csp/v22n10/21. pdf>. doi: 10.1590/S0102-311X2006001000028.

4. Kolling FL, Santos, JS. A influência dos fatores de risco nutricionais no desenvolvimento de câncer de mama em pacientes ambulatoriais do interior do Rio Grande do Sul, Brasil. Scientia Medica [Internet]. 2009 [acesso 2011 nov 27]; 19(3):115-21. Disponível em: <http://revistaseletronicas.pucrs.br/ ojs/index.php/scientiamedica/article/viewFile/4562/ 4264>.

5. Koide H, Oliveira PP, Rockenbach G, Di Pietro PF. Elaboração de material informativo sobre cuidados nutricionais para mulheres com câncer de mama. 
Rev Eletr Extensão [Internet]. 2010; 7(10):29-42 [acesso 2011 jul 2]. Disponível em: <http://www. periodicos.ufsc.br/index.php/extensio/article/ view/ 11394/16378 > doi: 10.5007/1807-0221.2010v7n $10 p 29$.

6. Brasil. Ministério da Saúde. Instituto Nacional de Câncer. Consenso Nacional de Nutrição Oncológica [Internet]. Rio de Janeiro: INCA; 2011. v.2 [acesso 2011 dez 28]. Disponível em: <http://www1.inca. gov.br/inca/Arquivos/consenso_nutricao_vol2. $\mathrm{pdf}>$.

7. Actis AM, Bergoc RM, Quartucci A, Outomuro D. Factores de riesgo convencionales y emergentes en cáncer de mama: un estudio en pacientes posmenopáusicas. Rev Chil Obstet Ginecol [Internet]. 2009 [acesso 2011 jul 10]; 74(3):135-42. Disponible em: <http://www.scielo.cl/scielo.php?script= sci_arttext\&pid=S0717-75262009000300002>. doi: 10.4067/S0717-75262009000300002.

8. Ziegler RG. Anthropometry and breast cancer. J Nutr. 1997; 127(5):924S-28S.

9. Padilha PC, Pinheiro RL. O papel dos alimentos funcionais na prevenção e controle do câncer de mama. Rev Bras Cancerol [Internet]. 2004 [acesso 2011 jul 30]; 50(3):251-60. Disponível em: <http:// jn.nutrition.org/content/127/5/924S.full.pdf>.

10. Cibeira GH, Guaragna RM. Lipídio: fator de risco e prevenção do câncer de mama. Rev Nutr. 2006; 19(1):65-75. doi: http://dx.doi.org/10.1590/\$141 5-52732006000100007.

11. Santos HS, Cruz WMS. A terapia nutricional com vitaminas antioxidantes e o tratamento quimioterápico oncológico. Rev Bras Cancerol [Internet]. 2001 [acesso 2011 jun 30]; 47(3):303-8. Disponível em: <http://www.inca.gov.br/rbc/n_47/v03/pdf/ artigo7.pdf>.

12. Basualdo DC. Câncer e agentes fitoquímicos nutricionais. Prática hospitalar [Internet]. 2009 [acesso 2011 nov 20]; 11(65):43-7. Disponível em: <http:// www.praticahospitalar. com.br/pratica\%2065/pdf/ 08.pdf>.

13. World Cancer Research Fund. American Institute for Cancer Research. Food, nutrition, physical activity, and the prevention of cancer: a global perspective. Washington (DC): AICR; 2007.

14. Doyle C, Kushi LH, Byers T, Courneya KS, DemarkWahnefried W, Grant B. Nutrition and physical activity during and after cancer treatment: an american cancer society guide for informed choices. CA-Cancer J Clin [Internet]. 2006 [cited $2011 \mathrm{Mar}$ 15]; 56(6):323-53. Available from: <http://caonline. amcancersoc.org/cgi/reprint/56/6/323>. doi: 10.33 22/canjclin.56.6.323.
15. World Health Organization. Obesity: preventing and managing the global epidemic. Geneva: WHO; 1998. Report of a WHO Consulation on Obesity.

16. Software de análise estatística. Epi Info. Versão 6.0 [programa de computador]. Atlanta: WHO; 1994.

17. Statistical Package for the Social Sciences. SPSS Statistics Base [computer program]. version 17.0 Chicago: SPSS.

18. Zart VB, Aerts D, Rosa C, Béria JB, Raymann BW, Gigante LP, et al. Cuidados alimentares e fatores associados em Canoas, RS, Brasil. Epidemiol Serv Saúde [Internet]. 2010 [acesso 2011 jul 15]; 19(2): 143-54. Disponível em: <http://scielo.iec.pa.gov.br/ pdf/ess/v19n2/v19n2a07.pdf>. doi: 10.5123/S167 9-49742010000200007.

19. Portilho F, Castañeda M, Castro IRR. A alimentação no contexto contemporâneo: consumo, ação política e sustentabilidade. Ciênc Saúde Coletiva [Internet]. 2011 [acesso 2011 jul 18]; 16(1):99-106. Disponível em: <http://redalyc.uaemex.mx/pdf/630/ 63015361010.pdf>. doi: 10.1590/S1413-812320 11000100014.

20. Brasil. Ministério da Saúde. Secretaria de Atenção à Saúde. Coordenação geral da política de alimentação e nutrição. Guia alimentar para a população brasileira: promovendo a alimentação saudável. Brasília: Ministério da Saúde; 2006.

21. Brasil. Ministério da Saúde. Instituto Nacional do Câncer. Prevenção e detecção do câncer [Internet]. Rio de Janeiro: INCA; 2011 [acesso 2011 jul 18]. Disponível em: <http://www.inca.gov.br/conteudo_ view.asp? $\mid \mathrm{D}=18>$.

22. Machado SP, Cavalcante MFM, Sabry MOD, Pinheiro LGP, Sampaio HAC. Idade e ingestão dietética de cálcio por mulheres sobreviventes de câncer de mama: um fator adicional de risco para o desenvolvimento da osteoporose? Rev Bra Cancerol [Internet]. 2007 [acesso 2011 jul 30]; 53(2):153-8. Disponível em: <http://www.inca.gov.br/rbc/n_53/ v02/pdf/artigo1.pdf>.

23. Kumar N, Allen KA, Riccardi D, Bercu BB, Cantor $A$, Minton $S$, et al. Fatigue, weight gain, lethargy and amenorrhea in breast cancer patients on chemoterapy. Is subclinical hypothyroidism the culprit? Breast Cancer Res Treat. 2004; 83(2):149-59. doi: 10.1023/B:BREA.0000010708.99455.e1d.

24. Kwan ML, Weltzien E, Kushi LH, Castillo A, Slattery ML, Caan BJ. Dietary patterns and breast cancer recurrence and survival among women with earlystage breast câncer. J Clin Oncol [Internet]. 2009 [cited 2011 Aug 15]; 27(6):919-26. Available from: <http://www.jcojournal.org/content/27/6/919. full>. doi: 10.1200/JCO.2008.19.4035. 
25. McEligot AJ, Largent J, Ziogas A, Peel D, AntonCulver $\mathrm{H}$. Dietary fat, fiber, vegetable, and micronutrients are associated with overall survival in postmenopausal women diagnosed with breast cancer. Nutr Cancer. 2006; 55(2):132-40. doi: 10.1207/s15327914nc5502_3.

26. Najas MS, Andreazza R, Souza ALM, Sachs $A$, Guedes ACB, Sampaio LR, et al. Padrão alimentar de idosos de diferentes estratos socioeconômicos residentes em localidade urbana da região sudeste, Brasil. Rev Saúde Pública [Internet]. 1994 [acesso 2011 ago 18]; 28(3):127-91. Disponível em: <http:// www.scielo.br/pdf/rsp/v28n3/04.pdf>. doi: 10.15 90/S0034-89101994000300004.

27. Santos JS, Costa COM, Nascimento Sobrinho CL, Silva MCM, Souza KEP, Melo BO. Perfil antropométrico e consumo alimentar de adolescentes de Teixeira de Freitas, Bahia. Rev Nutr [Internet]. 2005 [acesso 2011 ago 30]; 18(5):623-32. Disponível em: <http://www.scielo.br/scielo.php?pid=S1415-52 $732005000500005 \&$ script=sci_arttext\&tlng=pt>. doi: 10.1590/S1415-52732005000500005.

28. Oliveira SP, Thébaud-Mony A. Hábitos e práticas alimentares em três localidades da cidade de São Paulo (Brasil). Rev Nutr [Internet]. 1998 [acesso
2011 ago 18]; 11(1):37-50. Disponível em: <http:// www.scielo.br/scielo. php?pid=S1415-527319980 00100003\&script=sci_arttext>. doi: 10.1590/\$14 15-52731998000100003.

29. Fornés NS, Stringhini MLF. Development of a food frequency questionnaire and characterization of the food pattern consumption for low - income workers in the city of Goiânia, Goiás State State, Brazil. Acta Sci Health Sci [Internet]. 2005 [cited 2011 Dec 6]; 27(1):69-75. Available form: <http:// www.dietpro.com.br/site/components/com_ artigo/upload/54c0e3bfd040a1d11590793a42bb 156e.pdf>.

30. Sampaio HAC, Sabry MOD, Diniz DB, Sá MLB, Castro SMV, Feijão IEP et al. Consumo de frutas e hortaliças por indivíduos atendidos pelo Programa Saúde da Família na periferia da cidade de Fortaleza-Ceará. Rev APS [Internet]. 2010; 13(2):175-81. [acesso 2011 jun 13]. Disponível em:<http:// www.aps.ufjf.br/index.php/aps/article/viewArticle/ 455>.

Recebido em: 3/2/2012

Versão final em: 11/6/2012 Aprovado em: 11/7/2012 